\title{
Apuntes sobre el origen y la actualidad de las agencias de noticias
}

\author{
Commentaries about origin and present of news agencies
}

Erick Daniel Cruz-Mendoza ${ }^{1}$

\section{Resumen}

Las constantes transformaciones en la noción de agencias de noticias son el punto de partida para reflexionar acerca de su evolución y adaptabilidad de estas organizaciones ante las demandas del mercado informativo actual. Los usuarios buscan cada vez más noticias de una agenda temática particular que responda a sus necesidades y gustos. En este contexto, interesa conocer el origen de las agencias de noticias, su definición y clasificación para contrastar estos ítems con las particularidades de las agencias en la actualidad, con el propósito de reconocer a estos organismos en los sistemas informativos actuales.

Palabras clave: sistema de medios, especialización informativa, periodismo especializado, productores de información, corresponsales

\section{Abstract}

The constant transformations in the notion of news agencies are the starting point to reflect on their evolution and adaptability of these organizations to the demands of the current information market, in which users increasingly searching news from a particular thematic agenda that responds to your needs and tastes. In this context, it is interesting to know the origin of the news agencies, their definition and classification to contrast these items with the particularities of the "agencies" at present, with the purpose of recognizing these agencies in the current information systems.

Recibido: 18 de julio de 2019 Aceptado: 14 de noviembre de 2019 Publicado: 20 de diciembre de 2019

${ }^{1}$ Estudiante de la Maestría en Comunicación y Cultura Digital de la Facultad de Ciencias Políticas y Sociales en la Universidad Autónoma de Querétaro (México). Licenciado en Ciencias de la Comunicación por la Universidad del Mar (México) y diplomante en Divulgación Científica por el Centro Interdisciplinario de Investigación para el Desarrollo Integral Regional Unidad Oaxaca del Instituto Politécnico Nacional (México). Correo electrónico: cm.erick18@gmail.com 
Keywords: media system, information specialization, specialized journalism, information producers, correspondents

\section{Introducción}

Las noticias internacionales contribuyen a formar nuestro panorama sobre el mundo (Salinas, 1989), por esta razón es importante preguntarse ¿quién o quiénes producen esta información? En algunas ocasiones son los mismos medios de comunicación (prensa, radio y televisión) quienes envían a corresponsales a otros países para cubrir los acontecimientos de mayor trascendencia para el ámbito periodístico. Pero, ¿qué sucede cuando estos medios no tienen la capacidad de enviar a sus reporteros a otros países para elaborar las noticias? Supóngase que un periódico tiene en su nómina a 20 corresponsales y 10 enviados especiales. Esta treintena de trabajadores se encuentran en distintos puntos del mundo y se mueven constantemente para cubrir las noticias. Si cada uno se encarga de cubrir un país iquién cubre las noticias de los 163 países restantes?

Los sistemas de comunicación encargados de difundir diariamente información internacional se llaman agencias de noticias, "en la actualidad, sería inconcebible pensar en diarios y medios electrónicos de comunicación que no estuvieran vinculados a las agencias de noticias, pues éstas son piezas clave en el engranaje informativo" (Salazar, 1990: 11). Las agencias de noticias tienen su origen a mediados del siglo XIX en Europa en el contexto del surgimiento de los Estado-nación y la búsqueda de una identidad nacional en la cual fueron clave los medios de comunicación.

Fue en 1835 en Francia, cuando un exbanquero de nombre Charles Louis Havas visualizó el servicio de correspondencia de noticias como un negocio capaz de surtir a los diarios locales valiéndose de las palomas mensajeras. Así fue que "en ese momento nació la primera agencia de noticias en brindar un servicio diario con los sucesos relevantes de las principales ciudades europeas" (Botto, 2012: 21). Esta forma de concebir a las noticias como un producto de consumo necesario para el público dio paso a las agencias de noticias. A partir de aquel año surgieron otras agencias en diferentes países europeos y en Norteamérica, algunas fueron: New York Associated Press (Estados Unidos, 1848), Agencia Reuters (Inglaterra, 1851), Agencia Fabra (España, 1865), Associated Press (Estados Unidos, 1868), Agencia Telegráfica de San Petersburgo (Rusia, 1904), Agencia de noticias Nueva China (China, 1931), Deutsche Presse-Agentur (Alemania, 1949), entre otras. 


\title{
El origen: ¿qué son las agencias de noticias?
}

Artero, J. \& Morales, R. (2008) exponen el surgimiento de las agencias de noticias como una consecuencia del colonialismo, argumentan que el control militar y político fue determinante para la instauración de dichas entidades en los países subordinados de Francia y Alemania. A pesar de la fuerte identidad de las agencias hacia el país en donde surgieron, su servicio se ha caracterizado por la objetividad y la neutralidad.

\begin{abstract}
Aun en las agencias con fuerte identidad nacional, como la alemana Wolf, la francesa France Presse o la inglesa Reuters, la cobertura informativa de estos actores se ha caracterizado por la neutralidad, la objetividad y el intento de mostrar todos los aspectos de cualquier cuestión. Ese modelo de información en estado bruto, sin adjetivos, sin opiniones, sin complementos, indiferenciada, con el fin de ser objeto de utilización por los más diversos medios ha llevado a que su contenido haya sido considerado con frecuencia una commodity. (Artero, J. \& Morales, R., 2008: 54)
\end{abstract}

Sin embargo, existen casos de agencias de noticias acusadas de anteponer los intereses nacionalistas ante los informativos llegando al grado de situar a estas entidades como organizaciones propagandísticas de los Estados; Artero, J. \& Morales, R. (2008) citan el caso de la agencia EFE (España), la cual fue creada como sociedad anónima por parte de accionistas privados con la intención de que no fuera vista como un medio de información oficial.

Las agencias se posicionaron en la postguerra, durante la década de los setenta, cuando se desataron una serie de debates (de 1969 a 1986) entre los países del centro -liderados por Estados Unidos- y los países de la periferia -bajo el estandarte de la UNESCO. Allí se discutieron temas relacionados al flujo de información, la dependencia tecnológica y la propuesta final de un Nuevo Orden Mundial de la Información y Comunicación (NOMIC). A partir de entonces, las agencias ocupan un lugar importante en la agenda informativa debido a su capacidad de cobertura y a la velocidad de transmisión de contenidos, la cual funciona con base en Internet.

Desde la creación de la primera agencia hasta el día de hoy, estas organizaciones han cumplido la necesidad de ofrecer información externa más allá de los límites nacionales y regionales, además, se han caracterizado por ofrecer: urgencia (una agencia siempre lucha contra el tiempo), objetividad (sin opinión o juicio sobre la misma) y un servicio 
completo (difunden todo aquello que puede ser interesante para sus abonados y para las minorías).

Algunos autores, por mencionar a Muro (2006), consideran a las agencias como intermediarias de la información, pues, a diferencia de cualquier otro medio de comunicación no buscan noticias para sí mismas, sino para sus clientes. En este sentido Salazar señala "su actividad central se dirige hacia la búsqueda, el procesamiento y la difusión de la información en el menor tiempo posible" (1990: 51). Ahora bien, no existe un consenso respecto a la definición de las agencias de noticias, en cambio, hay propuestas para referirse a dichas organizaciones bajo otros nombres, por ejemplo, Ignacio Muro describe la evolución de las designaciones de las agencias:

Desde su origen han evolucionado, pero muy lentamente. Las diversas denominaciones que han recibido recogen ya cambios en su razón de ser y, también, connotan un alma en evolución: primero agencias de prensa, luego agencias de noticias, más tarde agencias de información, últimamente proveedoras de contenido. Diversas denominaciones que significan cosas parecidas y al mismo tiempo diversas, y que convienen entre ellas. (Muro, 2006: 60)

La polisemia de significados referentes a estas organizaciones es lo que lleva a plantearse qué se entiende por agencias de noticias hoy en día. Si bien es cierto, Muro reflexiona acerca de la evolución de estas en cuanto a la adaptabilidad de sus servicios a la lógica del mercado, otra interpretación se encuentra ligada a la transformación -sin evolución- de un proveedor de información dedicado a atender las demandas informativas de nichos específicos. Pasando a ser otro tipo de organización al no cumplir con el espíritu de las agencias tradicionales. ${ }^{2}$

Sobre las agencias de noticias en Latinoamérica, Aguiar (2015), traza una cronología de estas organizaciones responsables de la circulación de noticias en donde señala que la primera agencia de noticias creada en este contexto geográfico es la Agencia Americana Telegraphica (1847, Brasil). El autor observa que en la actualidad son varios los países que no cuentan con agencia estatal (Uruguay, de Chile, Colombia, Surinam, entre otros), lo cual es un obstáculo para la diversidad del escenario informativo, pues, al no contar con agencias propias otras las empresas periodísticas acaparan estos espacios,

\footnotetext{
${ }^{2}$ Una definición de <<agencias de noticias >> originada en el contexto mexicano, es la siguiente: "las agencias de noticias son empresas periodísticas proveedoras de información sobre los acontecimientos internacionales, regionales y locales más relevantes a los medios de comunicación - prensa, radio, televisión, internet-, además de a gobiernos e instituciones públicas y privadas, y que, al ampliar sus líneas de negocios, han incorporado como clientes o suscriptores a corporativos, compañías y organizaciones civiles (NOTIMEX, 2015: 13).
} 
por lo regular, las que lo hacen son compañías extranjeras y responden a convenios mercantiles entre los países. Al respecto, se espera que la creación de nuevas agencias estatales contribuya a emprender refuerzos en la cooperación Sur-Sur.

\section{Clasificación y operatividad de las agencias de noticias}

Las agencias se pueden clasificar de diferentes maneras: a partir del área geográfica en donde ofertan sus servicios, por el modelo económico de la organización o por el tema de información ofertado. A continuación, se proporciona un cuadro de la clasificación de las agencias con base en la información del libro Las agencias de noticias en la era digital. $^{3}$

Tabla 1

Clasificación de las agencias de noticias

\begin{tabular}{|c|c|c|c|}
\hline Categoría & Subcategoría & Definición & Ejemplos \\
\hline \multirow{2}{*}{ Geográfico } & Nacional & $\begin{array}{l}\text { Se incluyen aquellas cuyo radio de } \\
\text { acción se ubica en el país donde } \\
\text { tienen su sede. En general, recopilan } \\
\text { noticias dentro de su territorio y, del } \\
\text { mismo modo, distribuyen su } \\
\text { información en los medios de } \\
\text { comunicación del territorio } \\
\text { nacional. }\end{array}$ & $\begin{array}{l}\text { En México la agencia } \\
\text { Quadratín tiene el objetivo de } \\
\text { “abastecer de materiales } \\
\text { noticiosos, de opinión y } \\
\text { gráficos, a los medios de } \\
\text { comunicación de la entidad y } \\
\text { del país". }\end{array}$ \\
\hline & Mundial & $\begin{array}{l}\text { Se identifican por la amplitud y } \\
\text { perfeccionamiento de sus sistemas } \\
\text { de recopilación y distribución de } \\
\text { información en un gran número de } \\
\text { lenguas - alemán, árabe, español, } \\
\text { francés, inglés, portugués y ruso-, } \\
\text { por mantener delegaciones en más } \\
\text { de } 100 \text { países con miles de } \\
\text { empleados y corresponsales de }\end{array}$ & $\begin{array}{c}\text { Agence France Presse (AFP) de } \\
\text { Francia, Associated Press (AP) } \\
\text { y United Press International } \\
\text { (UPI) de Estados Unidos, } \\
\text { Reuters del Reino Unido, y } \\
\text { Tass, Itar-Tass, luego de la } \\
\text { desaparición de la Unión } \\
\text { Soviética. }\end{array}$ \\
\hline
\end{tabular}

\footnotetext{
${ }^{3}$ Los ejemplos citados pertenecen en su mayoría a la región de Iberoamérica, específicamente México, debido a la proximidad geográfica en donde se produjo esta investigación.
} 
Perspectivas Revista de Ciencias Sociales

ISSN 2525-1112 / Año 4 No. 8 Julio-Diciembre 2019, pp. 287-300

\begin{tabular}{|c|c|c|c|}
\hline & & tiempo completo y parcial. & \\
\hline & Internacional & $\begin{array}{l}\text { Estas agencias disponen de recursos } \\
\text { financieros y técnicos para la } \\
\text { recepción y transmisión de la } \\
\text { información y mantienen una red de } \\
\text { corresponsales ubicados en sitios } \\
\text { neurálgicos en ciertos países de los } \\
\text { cinco continentes, además cuentan } \\
\text { con clientes o suscriptores fuera de } \\
\text { su país de origen. }\end{array}$ & $\begin{array}{l}\text { Prensa Latina, de Cuba, y } \\
\text { NOTIMEX, de México, las } \\
\text { agencias españolas EFE y } \\
\text { Europa Press; además de la } \\
\text { agencia china Xinhua, y Kyodo } \\
\text { News, de Japón; la agencia de } \\
\text { noticias italiana ANSA } \\
\text { (Agenzia Nazionale Stampa } \\
\text { Associata); la agencia alemana } \\
\text { DPA (Deutsche Presse- } \\
\text { Agentur) e Inter Press Service } \\
\text { (IPS). }\end{array}$ \\
\hline & Regional & $\begin{array}{l}\text { Se caracterizan por extender sus } \\
\text { servicios a los países circunvecinos o } \\
\text { varias naciones del continente } \\
\text { donde tienen su sede. }\end{array}$ & $\begin{array}{l}\text { TASS concentró información } \\
\text { de las agencias pertenecientes a } \\
\text { la Unión Soviética durante la } \\
\text { Segunda Guerra Mundial. }\end{array}$ \\
\hline \multirow{3}{*}{$\begin{array}{l}\text { Modelo } \\
\text { económico }\end{array}$} & Privado & $\begin{array}{l}\text { Agencias cuyo modelo de negocios } \\
\text { es independiente, no } \\
\text { gubernamental, es decir, no } \\
\text { obtienen subsidio del estado. }\end{array}$ & $\begin{array}{c}\text { Associated Press (AP) - Estados } \\
\text { Unidos }\end{array}$ \\
\hline & Estatal & $\begin{array}{l}\text { Agencias cuyo presupuesto depende } \\
\text { del subsidio que otorga el estado. }\end{array}$ & Xinhua - China \\
\hline & Mixto & $\begin{array}{l}\text { Agencias cuyo modelo de negocios } \\
\text { no depende en su totalidad del } \\
\text { subsidio estatal y, por lo tanto, } \\
\text { cuenta con otros inversores. }\end{array}$ & EFE - España \\
\hline Información & General & $\begin{array}{l}\text { Agencias productoras de contenidos } \\
\text { diversificados, es decir, para cada } \\
\text { sección de los diarios. }\end{array}$ & $\begin{array}{l}\text { EFE, AP, AFP, Xinhua, IPS } \\
\text { (Inter Press Service), } \\
\text { NOTIMEX, entre otras. }\end{array}$ \\
\hline
\end{tabular}




\begin{tabular}{|l|l|c|c|}
\hline & Especializado & $\begin{array}{c}\text { Abordan temas determinados como } \\
\text { economía, deportes, espectáculos y } \\
\text { otros. En este ámbito se incluyen } \\
\text { compañías periodísticas. }\end{array}$ & $\begin{array}{c}\text { ESPN, Bloomberg, Tauropress, } \\
\text { Agencia de Prensa Científica } \\
\text { (España), Agence Presse } \\
\text { Medicale (Francia), entre otras. }\end{array}$ \\
\hline
\end{tabular}

Fuente: Elaboración propia con información de NOTIMEX, 2015: 21.

Para estructurar la información, las agencias de noticias emplean algunos subgéneros del periodismo tradicional: la nota, la entrevista y el reportaje. No obstante, también existen subgéneros periodísticos propios de las agencias, los cuales fueron creados con el objetivo de "captar mejor la complejidad de lo real y ofrecerlo al público. Esto implica dar a entender o persuadir introduciendo certidumbre, previsibilidad y orden" (Armañanzas citado en Rivas, 1999). Desde la perspectiva de Rivas, los géneros y subgéneros de las agencias de noticias son los siguientes:

- Informativos: Flash, Boletín, Urgente, Avance y Noticia.

- Interpretativos: Panorámica, Análisis y Crónica.

Desde su génesis, las agencias se han distinguido por producir y enviar información en bruto -sin opinión o juicio sobre la misma. A pesar de ello, en los últimos años ha crecido el interés por producir contenidos desde el género interpretativo, "la tendencia es general en todas las grandes [agencias] a aumentar el porcentaje de informaciones con firma, lo que implica un nivel más profundo de información, en cuanto crónicas o análisis" (Rivas, 1999: 66). Esto significa introducir géneros periodísticos que antes no se tomaban en cuenta para la redacción de materiales.

Las agencias también se caracterizan por ser organizaciones cerradas, es decir, no brindan información relacionada con su labor, "incluso son pocos los periodistas que conocen con cierta profundidad la estructura y el funcionamiento de estas organizaciones intermediarias de la información” (Salazar, 1990: 11). La información exteriorizada en sus sitios de Internet y en algunos libros no es suficiente para determinar la existencia de un único modelo de transmisión de las noticias. En cambio, esto hace pensar que cada agencia tiene una forma legítima para hacer llegar la información a sus clientes. Pedro Aguiar (2010) reconoce cuatro momentos durante los cuales las agencias de noticias se encargan de procesar la información para darle salida en cualquier formato y presentarla a los consumidores. 


\section{Corresponsales $\rightarrow$ Red Central $\rightarrow$ Centros Regionales $\rightarrow$ Clientes}

Este modelo da por hecho que el periodista -en este caso corresponsal- ha pasado por el proceso de recolección de información y su estructuración en un texto adecuado a los géneros/subgéneros de las agencias de noticias, por ello, inicia con el envío del material hacia las oficinas centrales para su publicación inmediata. Sin embargo, ¿qué sucede con las agencias de menor escala que no cuentan con centros regionales? o ¿qué sucede con las agencias en las cuales el corresponsal sube directamente la información al sitio de Internet y no tiene que enviarla a otras oficinas? Como se dijo, no se tiene la certeza de la existencia de un único modelo de procesamiento de la información, todo depende de la estructura y organización de la agencia de noticias.

También, se desconoce el dato exacto de la llegada de las agencias internacionales a México. Sin embargo, "el establecimiento de las agencias nacionales en nuestro país se dio tardíamente, cuando ya las extranjeras tenían acreditada su presencia en los medios locales" (Juárez, 1998: 9). La primera agencia en México se llamó INFORMEX y fue creada en 1961 como un negocio privado por Álvaro Gálvez y Fuentes. Por otro lado, se encuentra la Agencia Mexicana de Noticias (NOTIMEX) creada por el gobierno federal en 1968 con el objetivo de

ofrecer una información organizada y centralizada sobre los Juegos Olímpicos. Se ha dicho también que su creación, proyectada por el gobierno de Díaz Ordaz desde varios años antes, fue una respuesta al movimiento estudiantil, pero esta información no es muy sólida porque en agosto de aquel año el movimiento de los estudiantes apenas se iniciaba. (Trejo, 1989: 33)

Luego de caracterizar a las agencias de noticias se da pie a otra cuestión relacionada con dichas organizaciones. Desde hace décadas existe un debate en torno a la dependencia informativa ${ }^{4}$ en donde se identifica a cuatro agencias mundiales como las máximas proveedoras de información, conocidas como las Big Four, dentro de este grupo se encuentran la agencia americana Associated Press (AP), la inglesa Reuters, la francesa Agence France Presse (AFP) y United Press International (UPI) ${ }^{5}$.

\footnotetext{
${ }^{4}$ Este tema ha sido abordado por Raquel Salinas (1989), quien plantea a la dependencia como un problema para las agencias de América Latina.

${ }^{5}$ José Ángel Castro Savoie expone en su tesis doctoral titulada Las agencias transnacionales de prensa al final del siglo $X X$ (2002), que hoy en día se debe hablar de big three o tres grandes agencias, debido a que UPI se encontraba en proceso de desaparición.
} 
Para un mejor entendimiento, Hernández (2006) realizó un Reporte sobre las agencias internacionales de noticias y sindicalismo en América Latina, en el cual deja ver la dependencia de los medios de comunicación de algunos países como Brasil, Venezuela, Colombia, Uruguay, Costa Rica, República Dominicana, Argentina y México, entre otros, ante las agencias internacionales, la investigación reveló que:

En México opera un amplio grupo de agencias de noticias: las mexicanas, Agencia Mexicana de Investigación (AMI) y NOTIMEX, y las internacionales Reuters, EFE, Associated Press (AP), Agence France Press (AFP), United Press International (UPI), Inter Press Service IPS, ANSA, Deutsche Presse Agentur (DPA), Voller Ernst International Press, Xinhua News Agency, Yonhap News Agency, Prensa Latina, Europa Press, Agencia Getty, Bloomberg Bussiness News, Dow Jones Newswires, Houston Chronicle, Market News International, The Image Works, Kyodo News Service, Maghreb Arabe Press, Agencia Novosti, Itar-Tass, Agencia Latin Photo y la Agencia Vietnamita de Noticias (VNA). La mayoría de estas agencias internacionales, cuentan sólo con uno o dos corresponsales. (Hernández, 2006: 19)

El caso de México sirve para ilustrar la coexistencia de agencias de noticias en diversos ámbitos informativos, por ejemplo, el de la fotografía. Sin embargo, la dependencia informativa no sólo es cuestión de los países en desarrollo, en realidad, las agencias de noticias se expanden en diferentes regiones porque es parte de su lógica informativa al intentar cubrir las noticias de todo el mundo, el punto clave se encuentra en la incapacidad de las empresas nacionales por cubrir información dentro de sus fronteras y por ello, tienden a adquirir los productos de organizaciones ajenas a su nación que sí cuentan con el capital humano y la capacidad tecnológica para producir noticias fuera de su territorio.

\section{Agencias de noticias especializadas: trazando el camino}

En años recientes se ha dicho que el aumento de información "ocasiona la imposibilidad de aprehenderlo todo y de comprender lo que sucede en la realidad. A eso se añade el papel hegemónico de las nuevas tecnologías en la transmisión de información, la globalización y la expansión del conocimiento" (Delponti \& Pestano, 2012: 2). Por esta razón, hoy día la paradoja de la sobreinformación genera consumidores que demandan contenidos cada vez más selectos o específicos. Ante esto, 
los emisores de mensajes y canales de comunicación tienden a especializarse con el objetivo de atender las necesidades de los públicos emergentes.

De estas circunstancias nacen nuevos productores encargados de satisfacer necesidades informativas concretas. En el ámbito de las agencias también se dio una especialización de la información, por ejemplo, ¿quién hubiese imaginado una agencia de noticias dedicada únicamente a la producción de contenidos de la Tauromaquia? Es algo difícil de imaginar, sin embargo, desde 2001 un grupo de periodistas especializados en la información taurina decidieron acercar el mundo del toro a la nueva era de la información al crear la agencia de comunicación Tauropress. Este es un ejemplo del surgimiento de agentes productores de información selecta, puesta a disposición del público interesado.

En el escenario de la sobreinformación, desde el punto de vista de la oferta-demanda, los consumidores ya no tienen que esperar a que los diarios les sugieran una agenda de contenidos para leer, sino que, ahora, son ellos quienes escogen sus propios temas. Alvin Toffler (1999) avisó un cambio en la dinámica de la comunicación, la cual reconoce como personalizada, en donde el consumidor no se limita a esperar contenidos, ahora va por ellos y esto es posible gracias a la creciente oferta basada en la especialización del trabajo y por ende de los medios. Algo similar a lo que sucede actualmente con el análisis del Bigdata en función de la personalización de los contenidos, el cual está siendo incorporado por las industrias creativas, entre ellas las de contenidos noticiosos.

Lo anterior trae como consecuencias la reformulación de nuevos públicos, la adaptación de los medios tradicionales a la par del surgimiento de otros actores especializados en un tema y la ampliación de la oferta informativa.

[...] mientras Internet se convierte en el centro de los cambios en el ámbito periodístico y en el mercado de los medios, las agencias comienzan a desarrollar proyectos para adaptarse a las necesidades que impone informar en ese contexto, aunque estos proyectos todavía funcionan como apéndice de los negocios tradicionales, sin suficiente fuerza ni rasgos definidos en las agencias nacionales [...] La adaptación a las nuevas tecnologías impone al conjunto de las agencias la generación de información en múltiples soportes, y la supervivencia como proveedoras privilegiadas de contenidos dependerá de la rapidez con la que implementen los abruptos cambios que impone el sistema de medios. (Botto, 2012: 95-96)

Además de la generación de contenidos para múltiples soportes, la especialización temática de las agencias es una realidad para los consumidores, quienes se benefician de 
consultar información perteneciente a su tema de mayor interés con tan sólo acceder al portal de Internet de la agencia especializada.

Con esto se reafirma la tesis de Toffler (1999) acerca del cambio en la dinámica de la comunicación. Ahora los consumidores demandan los contenidos y no esperan para verlos a que lleguen a sus manos en forma de diarios o suplementos. Gracias al Internet brincan a los intermediarios y llegan directamente a los proveedores de información. Sólo por mencionar un ejemplo, el diario mexicano Reforma (s. f.) en su sitio de Internet tiene algunas noticias del día, sin embargo, para leer los textos por completo se debe pagar una membresía de aproximadamente $\$ 150.00$ mensuales para consultar los materiales en digital a través de la página de Internet y las aplicaciones para smartphones. Este ejercicio no representa el consumo gratuito de la información, en algunos casos existen restricciones de pago, lo cual marca una diferencia entre el lector "gratuito" con acceso al 20\% de la información y el lector "de paga" con acceso al 100\% de los contenidos, por decir un guarismo.

Sin duda los cambios tecnológicos, la especialización de la información y el surgimiento de actores dedicados a la producción de contenidos también han acarreado consecuencias negativas para los consumidores, por ejemplo, el caso de las fake news (noticias falsas) como herramienta para falsear información en la era digital. Muñoz explica que desde siempre han existido las informaciones fabricadas, pero nunca a la escala suscitada en los últimos años, "en 2016 se inscribieron 43 sitios de Internet, los que publicaron más de 750 Fake News sólo en Estados Unidos que tuvieron un amplio compromiso -compartir, likes, comentarios- en redes sociales, particularmente en la plataforma Facebook" (Muñoz, 2017: 36). Siguiendo la idea del autor, este tipo de noticias tienen cabida en las audiencias porque parecen verdaderas, es decir, apelan a los marcos contextuales, a la identidad de los lectores y a los valores de los mismos, de ahí proviene la clave de su éxito. En contraparte, las empresas y los gobiernos trabajan en la regulación de este problema, sin embargo, para Muños (2017) los periodistas tienen la responsabilidad de desmentir inmediatamente este tipo de contenidos, pues, contribuyen a la formación incorrecta de la opinión pública.

\section{Reflexiones finales}

A manera de síntesis, se pueden traer a cuentas varias reflexiones acerca del andar de las agencias de noticias. Primero, el espíritu de suministrador de noticias se está transformando a uno de suministrador de contenidos, en donde tienen cabida las 
noticias. No obstante, las organizaciones informativas se están enfrentando a nuevos modelos de negocio para solventar su producción. Así lo ha hecho ver Muro (2006) quien apunta a que estas deben de diversificar su oferta de noticias a una más amplia de contenidos: video-columna, foto-reportaje, infografía, entre otros varios propios de la comunicación digital.

De esta manera, las agencias de noticias ya no pueden seguir concibiéndose únicamente como proveedoras de noticias, sino, estas deben echar mano de las estrategias del mercado digital para encontrar un lugar en este ambiente y desde ahí continuar con la distribución de contenidos. Sobre los formatos tradicionales, estos son escuetos si se piensan en la dinámica de las pantallas digitales mediante las cuales se consumen contenidos noticiosos en la actualidad. Aquí también hay que voltear a ver lo que están haciendo otros proveedores de contenidos para recuperar la estructura y el fondo de manera provechosa.

Para concluir este trabajo, es preciso poner énfasis en la actividad de los periodistas, quienes son responsables de producir información objetiva y veraz con el propósito de contribuir al desarrollo informativo de las sociedades. Las agencias y en general, los medios de comunicación tienen un carácter humano en el cual descansan las noticias, es decir, estas no se encuentran tiradas en el suelo y los periodistas simplemente las recogen y publican. Aunque no lo parezca, el proceso de la elaboración de noticias es todavía más complejo y cada organización tiene sus métodos y herramientas para lograrlo y, más aun, cuando se atiende a las necesidades informativas en el entorno digital.

\section{Bibliografía}

Aguiar, P. (2010). Agências de Noticias e Sistemas Internacionais de Informação: do pool nãoalinhado à comunicação em redes. Tesis de Maestría no publicada, Brasil: Universidade Federal do Rio de Janeiro.

Aguiar, P. (2015). Agencias de Noticias de América Latina en el siglo XXI: informe preliminar de investigación. En ULEPICC (7 al 11 de diciembre de 2015). Anales del IX Congreso de la Unión Latina de la Economía Política de la Información, la Comunicación y la Cultura (ULEPICC). Disponible en https://www.academia.edu/19676560/Agencias_de_Noticias_de_Am\%C3\%A9ri ca_Latina_en_el_siglo_XXI_informe_preliminar_de_investigaci\%C3\%B3n

Artero, J. \& Morales, R. (2008). Opciones estratégicas de las agencias de noticias europeas: Reuters, France Presse y EFE. Comunicación y Sociedad, 1, 53-79. 
Disponible

https://www.unav.es/fcom/communicationsociety/es/articulo.php?art_id=37

Botto, M. (2012). Historia de las agencias de noticias. Desde su creación hasta el período de entreguerras. Buenos Aires, Argentina: Academia Nacional de Periodismo. Disponible en http://www.academiaperiodismo.org.ar/publicaciones/agenciasdenoticiasweb. pdf

Delponti, M. \& Pestano, J. (2012). El papel del periodismo cultural en la construcción simbólica de un imaginario social globalizado. En Feliu, E. (Coord.). Comunicación: Memoria, Historia y Modelos, 296-302. España: Foro Universitario de Comunicación. Jornadas.

Hernández, R. (2006). Reporte sobre las agencias internacionales de noticias y sindicalismo en América Latina. Brasil: Federação Nacional dos Jornalistas (FENAJ).

Juárez, O. (1998). El trabajo del reportero en una agencia de noticias. Tesis de licenciatura no publicada, Universidad Nacional Autónoma de México, México. Disponible en http://132.248.9.195/pdbis/268612/Index.html

Muñoz, P. (2017). Medios de comunicación y posverdad: Análisis de las noticias falsas en elecciones presidenciales de EE. UU. de 2016. Tesis de Máster no publicada. Universidad Autónoma de Barcelona, España. Disponible en https://ddd.uab.cat/pub/trerecpro/2017/hdl_2072_293813/TFM_Priscilla_Mu noz.pdf

Muro, I. (2006). Globalización de la información y agencias de noticias: entre el negocio y el interés general. España: Paidós.

NOTIMEX. (2015). Las agencias de noticias en la era digital. México: Biblioteca Mexicana del Conocimiento.

Reforma. (s. f.). Suscripciones [versión digital]. Disponible en https://secure.gruporeforma.com/reforma/suscribete/?utm_campaign=include _suscripcio

Rivas, J. (1999). Géneros periodísticos en las agencias de prensa. Estudios sobre el mensaje periodístico, 5, 159-167. Disponible en https://revistas.ucm.es/index.php/ESMP/article/viewFile/ESMP9999110159A $/ 12991$

Salazar, H. (1990). Las agencias de noticias en América Latina: estructura y funcionamiento. México: Editorial Trillas. 
Salinas, R. (1989). No es fácil romper la dependencia: el caso de las noticias. Diálogos de la Comunicación, 24. Disponible en http://dialogosfelafacs.net/wpcontent/uploads/2015/24/24-revista-dialogos-no-es-facilromper-dependenciaen-las-noticias.pdf

Toffler, A. (1999). La tercera ola (Trad.). España: Sudamericana. (Trabajo original publicado en 1980).

Trejo, D. (1989). Las agencias de información en México. México: Editorial Trillas. 